\title{
Analysis of Atmospheric Transmission Characteristics on the Bit Error Rate of Atmospheric Laser Communication System
}

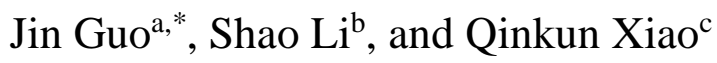 \\ ${ }^{a}$ School of Electronic and Information Engineering, Xi'an Technological University, Xi'an, 710021, China \\ ${ }^{b}$ China North Vehicle Research Institute, Bei Jing. 100072, China \\ ${ }^{c}$ School of Electronic and Information Engineering, Xi'an Technological University, Xi'an, 710021, China
}

\begin{abstract}
The atmospheric transmission characteristics influence the information transmission of atmospheric laser communication system, increase the bit error rate, and reduce the information transmission reliability. Under the atmospheric attenuation effect, according to the relation of atmospheric transmittance and transmission distance and atmospheric visibility, this paper establishes the calculation model of bit error rate. Under the influence of atmospheric turbulence, in accordance with restricted relationship between the probability density of light intensity and the atmospheric turbulence intensity, a model of bit error rate is built. When atmospheric attenuation effect and atmospheric turbulence effect take up \different weights in the atmospheric channel, by analysing the influence of atmospheric attenuation and atmospheric turbulence to bring about the bit error rate, a new model of bit error rate in atmospheric laser communication system is constructed. Through calculation and analysis, the results show that the main factor of influence of bit error rate is atmospheric turbulence effect; meanwhile, the impact of atmospheric attenuation is not negligible.
\end{abstract}

Keywords: atmospheric laser communication system; atmospheric transmission characteristics; bit error rate; transmission distance

(Submitted on March 2, 2018; Revised on April 9, 2018; Accepted on May 20, 2018)

(C) 2018 Totem Publisher, Inc. All rights reserved.

\section{Introduction}

Laser has the characteristics of high brightness, strong directivity, good monochromatic, and strong coherence. The laser communication system transmits information by laser; the transmission medium of laser communication system is atmosphere, so the laser communication system is known as the atmospheric laser communication system. The atmospheric laser communication system has many advantages: the multi-channel data and image can transmit simultaneously because of the small divergence angle of laser beam and the good directivity, and the structure of communication equipment is light and cheap. At the same time, it also brings some problems, such as: the communication equipment must have the better stability and the higher precision when the direction of laser is strong, the operation of communication equipment is complicated owing to the transmission and receiver of aiming is difficult, and the climate affects the quality of communication and causes the communication disruptions under severe weather. In addition, the atmospheric molecule has oxygen, nitrogen, carbon dioxide and water vapor, owing to the atmospheric molecule absorbing light signal and that the atmospheric molecular density is uneven; the atmospheric molecule brings about light scattering. The suspended of dust, smoke, ice crystals, salt particles, microorganism and tiny water droplets also lead to light scattering in the atmosphere; therefore, the cloud, rain, fog, snow cause laser's power attenuated severely while bringing about the atmospheric attenuation effect. The air convection on the earth's surface leads to atmospheric turbulence; the atmospheric turbulence causes laser beam deflection, beam diffusion, beam flicker and jitter emerge in the laser transmission. We should consider the bit error rate by atmospheric attenuation effect and atmospheric turbulence effect in atmospheric laser communication system.

At present, the atmospheric attenuation brings different atmospheric visibility, and the aperture size of detection receiver and the atmospheric visibility affect the bit error rate of wireless optical communication system [1]. The bit error rate of

\footnotetext{
* Corresponding author.

E-mail address: guojin_1980@126.com
} 
atmospheric optical communication system is related to the signal to noise ratio of detector, the diameter of receiving aperture, and the variance of fluctuation of light intensity, owing to atmospheric turbulence [3]. Atmospheric turbulence affects the bit error rate of wireless optical radio frequency communication system [6]. The different scale of atmospheric turbulence brings about the variable bit error rate in the laser communication system using multi-beam transmission technology [2]. Atmospheric turbulence leads to the flicker of laser intensity to affect the performance of laser communication system. The changing intensity of atmospheric turbulence and the varied wavelength of laser bring about the different bit error rate of laser communication system [7]. In order to scientifically analyze bit error rate of atmospheric laser communication system, this paper considers atmosphere attenuation and atmospheric turbulence that influence the transmission of atmospheric laser communication system, and deduces a new model of bit error rate of atmospheric laser communication system.

\section{The Model of Atmospheric Transport Channel}

Laser is the key to communication through atmosphere in the laser communication system. The transmission characteristics and laws of laser directly affect the quality of atmospheric laser communication. The study shows that atmospheric attenuation effect, atmospheric turbulence effect and nonlinear optical effect are important factors for the laser atmospheric transmission [8]. The atmospheric attenuation refers to the loss of laser's energy due to atmospheric absorption and atmospheric scattering; the loss of laser's energy affects transmission distance and reliability [9]. The atmospheric turbulence is the vortex flow in the atmosphere; the vortex flow makes air velocity, temperature and refractive random fluctuate in time and space. It also causes the coherence of laser beam poor due to receiving light flashing and light drifting [4]. The nonlinear optical effect is the effect of strong laser transmission in the atmosphere. This paper focuses on atmospheric attenuation and atmospheric turbulence, because the atmospheric laser communication system transmits information without using strong laser.

\subsection{The Model of Atmospheric Attenuation Effect}

The attenuation of laser's power mainly comes from the absorption and scattering of atmospheric molecules and aerosol particles when the laser transmits horizontally through lower atmosphere in the laser communication system. Assuming that laser's wavelength is the wavelength of atmosphere communication window, we can ignore the absorption of atmospheric molecular caused by attenuation of laser's power. If the laser's wavelength is wavelength of near infrared band, the Rayleigh scattering coefficient of near infrared band is very small; we can omit the atmospheric molecular scattering bringing about attenuation of laser's power. The main component of aerosol particles is haze, and its absorption of laser's energy is very small. we can ignore absorption of aerosol particles caused by attenuation of laser's power. Therefore, we only research the scattering of aerosol particles produced the attenuation of laser's power in the lower atmosphere; the scattering is also known as the Mie scattering. In addition, the inhomogeneity of atmospheric density causes attenuation of laser's energy. With the increment of transmission distance, the more the attenuation of laser's energy, the atmospheric visibility and the transmission distance and the wavelength of laser affect the atmospheric transmittance; the atmospheric transmittance is expressed in formula (1).

$$
\tau_{0}=\exp \left(\frac{-3.912 L}{V}\left(\lambda / \lambda_{1}\right)^{n}\right)
$$

In (1), $L$ is the transmission distance, $V$ is the atmospheric visibility, $\lambda$ is the wavelength of laser, $n$ is the degree of dependence on the wavelength of light that Mie scattering brings about attenuation of optical signal, also call the correction factor, $\lambda_{1}=600 \mathrm{~nm}$.

Because the difference of atmospheric visibility influences the size of correction factor, the calculation model of atmospheric transmittance is more scientific by using the model of correction factor from Kim [10]. The specific model of correction factor is shown as formula (2).

$$
n=\left\{\begin{array}{cc}
1.6 & V>50 \mathrm{~km} \\
1.3 & 6 \mathrm{~km}<V \leq 50 \mathrm{~km} \\
0.16 V+0.34 & 1 \mathrm{~km}<V \leq 6 \mathrm{~km} \\
V-0.5 & 0.5 \mathrm{~km}<V \leq 1 \mathrm{~km} \\
0 & V<0.5 \mathrm{~km}
\end{array}\right.
$$

Assume that the laser beam turns into the Gaussian beam through the atmosphere. Because of the atmospheric attenuation, the intensity of laser beam changes when the laser beam reaches the photoelectric detector of receiving end; the receiving 
intensity of photoelectric detector is expressed in formula (3).

$$
I_{1}=I_{0} \cdot \tau_{0} \cdot \exp \left(-\frac{2 h}{0.5 X}\right)
$$

In (3), $I_{0}$ is the laser emission intensity, $h$ is the radial distance from laser axis, and $0.5 X$ is the radial position that the light intensity is $1 / e^{2}$ in the laser axis.

The responsivity of photoelectric detector changes in the atmospheric laser communication system because the high laser's power illuminates photoelectric detector, or irradiation time is too long on photoelectric detector. These factors lead to melt the interior material of photoelectric detector, and make a large number of carrier breakdown photoelectric detectors. Field effect produces carrier, which eventually causes local damage of photoelectric detector, and reduces the responsivity of photoelectric detector. Therefore, in order to all-weather and work stably and reliably of atmospheric laser communication system, we select the laser's power as less than the damage threshold of photoelectric detector, which ensures the responsivity of photoelectric detector is a given responsively. The receiving laser intensity of photoelectric detector and the response degree of photoelectric detector affect the output voltage of photoelectric detector; the output voltage of photoelectric detector can be calculated by equation (4).

$$
U_{d}=I_{0} \cdot R \cdot \exp \left(\frac{-3.912 L\left(\lambda / \lambda_{1}\right)^{n}}{V}-\frac{4 h}{X}\right)
$$

In (4), $R$ is the given response degree of photoelectric detector.

In atmospheric laser communication system, the noise of photoelectric detector mainly comes from thermal noise. The internal electrons of an object above freezing generate thermal noise by the non-regular thermal motion; $I_{T}^{2}$ is its variance; $I_{T}^{2}=\frac{4 K_{B} T B W}{R_{T}} . K_{B}$ is the Boltzman constant [11]; its value is $1.38 \times 10^{-34} \mathrm{~J} / \mathrm{K} . T$ is the equivalent noise temperature, $B W$ is the bandwidth of detector's subsequent filter, and $R_{T}$ is load impedance. Assume that the output signal to noise ratio of photoelectric detector is the ratio of the output voltage of photoelectric detector to the mean value of noise voltage; it can be expressed by (5).

$$
S N R_{1}=U_{d} /\left(I_{T} \cdot R_{T}\right)
$$

In order to obtain the relation of the laser emission intensity, the given responsivity of photoelectric detector and the transmission distance, combine formulas (4) and (5). The signal to noise ratio of photoelectric detector is described in formula (6).

$$
S N R_{1}=\frac{I_{0} \cdot R \exp \left(\frac{-3.912 L\left(\lambda / \lambda_{1}\right)^{n}}{V}-\frac{4 h}{X}\right)}{\sqrt{4 K_{B} T B W R_{T}}}
$$

The output voltage of photoelectric detector, the mean square value of noise voltage, and the output signal to noise ratio of photoelectric detector affect the bit error rate of atmospheric laser communication system; the bit error rate model can be expressed by (7).

$$
B E R_{1}=0.5 \operatorname{erfc}\left(\frac{I_{0} \operatorname{Rexp}\left(\frac{-3.912 L\left(\lambda / \lambda_{1}\right)^{n}}{4 \sqrt{2} \sqrt{K_{B} T B R_{T}}}-\frac{4 h}{X}\right)}{4}\right)
$$

Considering that the atmospheric attenuation brings about bit error rate of atmospheric laser communication system, we 
can find that the transmission distance, the atmospheric visibility, and the laser intensity influence the bit error rate.

\subsection{The Model of Atmospheric Turbulence Effect}

The atmospheric turbulence makes the air density of atmosphere randomly fluctuate, while the refractive index of atmosphere affects the air density. So, the refractive index of atmosphere also varies with the change of space and time without rules. It is defined as the atmospheric turbulence effect. We use the single beam of laser to transmit signal in atmospheric laser communication system; the atmospheric turbulence effects the transmission of laser beam, and makes the light emerge flashing in the atmospheric transmission. The probability density function of laser intensity can be expressed by (8).

$$
P(I)=\frac{\exp \left(-\frac{1}{8 \sigma_{s}^{2}}\left(\ln \left(I / I_{0}\right)+2 \sigma_{s}^{2}\right)^{2}\right)}{2 \sqrt{2 \pi} \sigma_{s} I}
$$

In (8), $I$ is the light intensity of the receiving photoelectric detector. $\sigma_{s}^{2}$ is the logarithmic variance of fluctuation of laser intensity; it can indicate the fluctuation degree of laser intensity.

If the laser beam is a plane wave, the transmission of laser beam is horizontal, and the logarithmic variance of fluctuation of laser intensity can be acquired by (9).

$$
\sigma_{s}^{2}=0.31 C_{n}^{2} k^{7 / 6} L^{11 / 6}
$$

In (9), $C_{n}$ is the atmospheric refractive index, $k$ is the optical wave number, and $k=2 \pi / \lambda[5,12]$.

The weak atmospheric turbulence affects the communication quality of atmospheric laser communication system. We combine formula (8) with (9). Consider the weak atmospheric turbulence; the probability density function of laser intensity can be described by (10).

$$
P(I)=\frac{\exp \left(-\frac{\ln \left(I / I_{0}\right)\left(\ln \left(I / I_{0}\right)+1.24 C_{n}^{2} k^{7 / 6} L^{11 / 6}\right)}{2.48 C_{n}^{2} k^{7 / 6} L^{11 / 6}}\right)}{2.79 I C_{n} k^{7 / 12} L^{11 / 12} \cdot \exp \left(0.15 C_{n}^{2} k^{7 / 6} L^{11 / 6}\right)}
$$

The atmospheric turbulence effect causes random fluctuation of atmospheric refractive index and makes the scintillation of receiving optical signal. We think that the atmospheric laser communication system brings about random noise, and increases bit error rate. The logarithmic intensity fluctuation is shown in formula (11).

$$
\omega=\ln \frac{I}{I_{0}}=\ln \left(\frac{\Gamma_{0}+\Gamma_{1}}{\Gamma_{0}}\right)=\ln (1+\varepsilon)
$$

In (12), $\Gamma_{0}$ is the amplitude of light wave without atmospheric turbulence, $\Gamma_{1}$ is the amplitude of noise with atmospheric turbulence, and $\varepsilon$ is the ratio of the amplitude of noise to the amplitude of signal. $\varepsilon=\Gamma_{1} / \Gamma_{0}$.

The atmospheric turbulence influences the atmospheric laser communication system. The signal to noise ratio of receiving photoelectric detector is the ratio of the laser emission intensity to the average intensity of noise; the signal to noise ratio can be written as formula (12).

$$
S N R_{2}=\frac{I_{0}}{I_{2}}=\frac{\Gamma_{0}^{2}}{\Gamma_{1}^{2}}=\frac{1}{\varepsilon^{2}}
$$

In (12), $I_{2}$ is the average intensity of noise in the atmospheric laser communication system. 
In the case of weak atmospheric turbulence, the noise is relatively small. Assume that $\omega=\ln (1+\varepsilon)=\varepsilon$, because of $\omega^{2}=\sigma_{s}^{2}, \varepsilon^{2}=\sigma_{s}^{2}$, combine formulas (9) and (12). The signal to noise ratio can be shown as formula (13).

$$
S N R_{2}=\frac{1}{0.31 C_{n}^{2} k^{7 / 6} L^{11 / 6}}
$$

Assume that the atmospheric laser communication system is the OOK intensity modulation; the system noise is the zero mean Gaussian white noise, and the bit error rate is gained by formula (14).

$$
B E R_{2}=0.5 \int_{0}^{\infty} P(I) \operatorname{erfc}\left(\frac{S N R_{2}}{2 \sqrt{2}}\right) d I
$$

The bit error rate is related to the transmission distance and the atmospheric visibility and the laser intensity. Formula (15) combines formulas (8), (13) and (14).

$$
B E R_{2}=\frac{\operatorname{erfc}\left(\frac{1}{0.11 C_{n}^{2} k^{7 / 6} L^{11 / 6}}\right)}{5.58 C_{n} k^{7 / 12} L^{11 / 12} \cdot \exp \left(0.15 C_{n}^{2} k^{7 / 6} L^{11 / 6}\right)} \cdot \int_{0}^{\infty} \exp \left(-\frac{\ln \left(I / I_{0}\right)\left(\ln \left(I / I_{0}\right)+1.24 C_{n}^{2} k^{7 / 6} L^{11 / 6}\right)}{2.48 C_{n}^{2} k^{7 / 6} L^{11 / 6}}\right) / I d I
$$

\section{The Model of Bit Error Rate of Atmospheric Laser Communication System}

In the atmospheric laser communication system, laser generates the scattering of aerosol particles under the transmission of lower atmosphere. The scattering of aerosol particles causes attenuation of laser's power in the atmospheric transmission. With the increment of transmission distance and the attenuation of laser's power exponential decay, this is atmosphere attenuation effect. The atmospheric turbulence causes the refractive index of atmosphere to vary with space and time without rules, forming the atmospheric turbulence effect. The atmospheric turbulence effect causes the light wave to fluctuate in the atmospheric transmission, making the laser intensity's fluctuate. The attenuation of laser's power and the fluctuation of laser intensity affect bit error rate; therefore, we must concern the atmospheric attenuation effect and atmospheric turbulence effect. The new model of bit error rate can be written as formula (16).

$$
B E R_{\text {total }}=\alpha B E R_{1}+\beta B E R_{2}
$$

In (16), $\alpha$ is the weighting of atmospheric attenuation effect that laser transmit in the atmosphere, and $\beta$ is the weighting of atmospheric turbulence effect that laser transmit in the atmosphere. $\alpha+\beta=1$.

In order to comprehensively analyze the bit error rate of atmospheric laser communication system, combine formulas (7), (15) and (16); formula (17) shows the result.

$$
\begin{aligned}
& B E R_{\text {total }}=0.5 \operatorname{\alpha erfc}\left(\frac{I_{0} \operatorname{Rexp}\left(\frac{-3.912 L\left(\lambda / \lambda_{1}\right)^{n}}{V}-\frac{4 h}{X}\right)}{4 \sqrt{2} \sqrt{K_{B} T B R_{T}}}\right)+ \\
& 0.09 \beta \frac{\operatorname{erfc}\left(\frac{1}{0.11 C_{n}^{2} k^{7 / 6} L^{11 / 6}}\right)}{C_{n} k^{7 / 12} L^{1 / 12} \cdot \exp \left(0.15 C_{n}^{2} k^{7 / 6} L^{1 / 6}\right)} \cdot \int_{0}^{\infty} \exp \left(-\frac{\ln \left(I / I_{0}\right)\left(\ln \left(I / I_{0}\right)+1.24 C_{n}^{2} k^{7 / 6} L^{11 / 6}\right)}{2.48 C_{n}^{2} k^{7 / 6} L^{1 / 6}}\right) / I d I
\end{aligned}
$$

From (17), it can be seen that bit error rate is related to transmission distance, atmospheric visibility, laser intensity, atmospheric structure constant, and te wavelength of laser. 


\section{Calculation and Analysis}

In the atmospheric laser communication system, in order to keep the laser's wavelength in the atmospheric window, assume that the laser's wavelength is 1 to $2 \mu \mathrm{m}$. The responsivity of photoelectric detector is $10^{-7} \mu \mathrm{s}$. The highest laser's power is $10 \mathrm{~W}$, and the weak atmospheric turbulence intensity is $1 \times 10^{-15}$ to $5 \times 10^{-15} \mathrm{~m}^{-2 / 3}$. We calculate and analyze the relation of bit error rate, atmospheric visibility and transmission distance, and laser intensity under atmospheric attenuation effect. We obtain and analyze the relation of bit error rate, atmospheric structure constants, and laser's wavelength under atmospheric turbulence effect. Considering atmospheric attenuation effect and atmospheric turbulence effect in the atmospheric laser communication system, we analyze the rule of bit error rate.

According to equation (7), we find that atmospheric visibility and transmission distance and laser intensity affect the bit error rate under atmospheric attenuation. Figure 1 shows the relationship between bit error rate and transmission distanc when atmospheric visibility is $0.4 \mathrm{~km}, 3 \mathrm{~km}, 6.5 \mathrm{~km}$ respectively. Figure 2 analyzes the relation of bit error rate and transmission distance when the laser intensity is $658 \mathrm{~W} / \mathrm{cm}^{2}, 804 \mathrm{~W} / \mathrm{cm}^{2}, 937 \mathrm{~W} / \mathrm{cm}^{2}$ respectively.

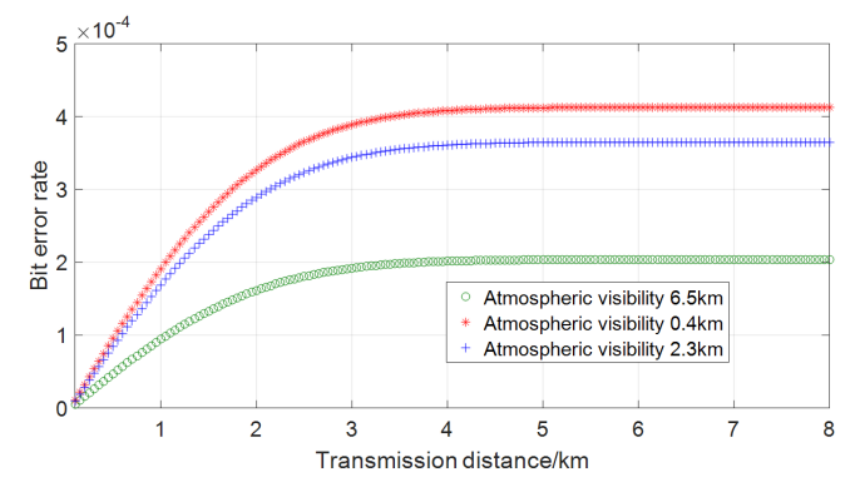

Figure 1. The curve of bit error rate and transmission distance under different atmospheric visibility

It can be seen from Figure 1 that the longer the transmission distance, the higher the bit error rate; otherwise, the lower the bit error rate. The transmission distance of atmospheric laser communication system is unchanged; the greater the atmospheric visibility, the lower the bit error rate. When atmospheric visibility is $6.5 \mathrm{~km}$, the transmission distance is $5 \mathrm{~km}$ and the bit error rate is about $2 \times 10^{-4}$.

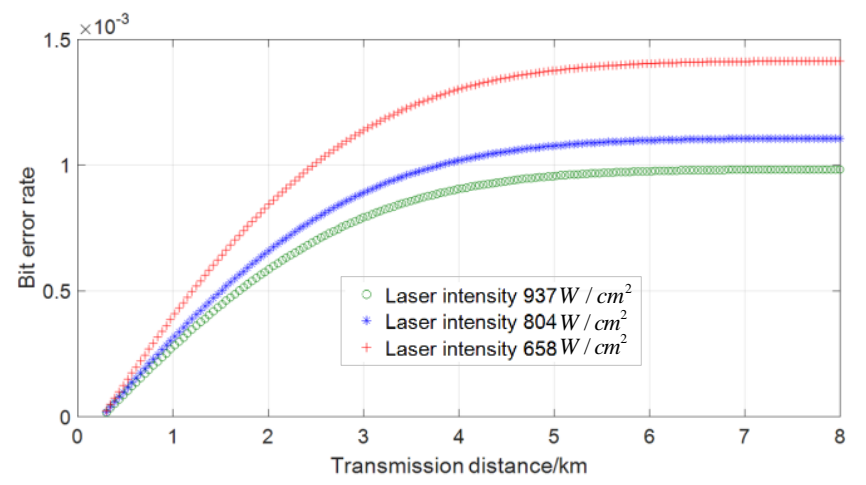

Figure 2. The curve of bit error rate and transmission distance under different laser intensity

As seen from Figure 2, with the increment of laser intensity, the bit error rate gradually decreases. In the case of ensuring a certain bit error rate of the atmospheric laser communication system, the higher the laser intensity, the longer the transmission distance. When laser intensity is $937 \mathrm{~W} / \mathrm{cm}^{2}$, the transmission distance is $5 \mathrm{~km}$ and bit error rate is about $1 \times 10^{-3}$.

Thus, if we only focus on the affect of atmospheric attenuation, when the transmission distance is $5 \mathrm{~km}$, the smaller the laser intensity and the bigger the bit error rate. In order to reduce the bit error rate, increase the laser emission power to achieve the purpose of improving laser intensity.

Based on formula (15), we discover that atmospheric turbulence intensity, laser's wavelength and transmission distance 
influence the bit error rate under atmospheric turbulence. If the atmospheric turbulence intensity is $1.2 \times 10^{-15} \mathrm{~m}^{-2 / 3}$, $2.5 \times 10^{-15} \mathrm{~m}^{-2 / 3}, 4.6 \times 10^{-15} \mathrm{~m}^{-2 / 3}$ respectively, we gain the curve of bit error rate and transmission distance under different atmospheric turbulence intensity, as shown in Figure 3. If laser's wavelength is $1.2 \mu \mathrm{m}, 1.5 \mu \mathrm{m}, 1.8 \mu \mathrm{m}$ respectively, we calculate the curve of bit error rate and transmission distance under different laser's wavelength, as shown in Figure 4 . $C_{n}$ expresses the atmospheric turbulence intensity in Figure 3.

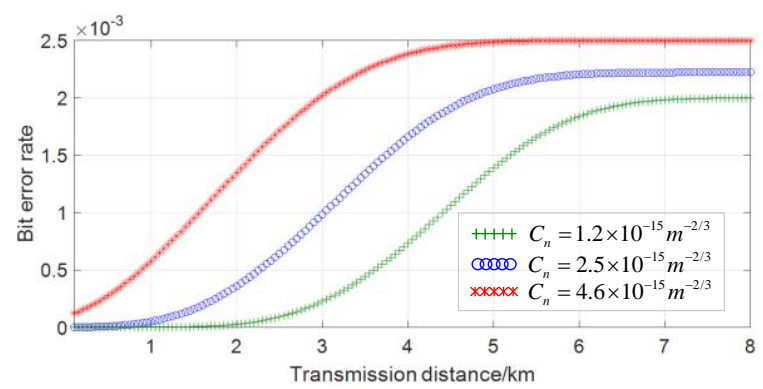

Figure 3. The curve of bit error rate and transmission distance under different atmospheric turbulence intensity

Figure 3 shows that bit error rate increases with the increase of transmission distance; the larger the atmospheric turbulence intensity, the bigger the bit error rate. When atmospheric turbulence intensity is $1.2 \times 10^{-15} \mathrm{~m}^{-2 / 3}$, the transmission distance is $5 \mathrm{~km}$, bit error rate is about $1.4 \times 10^{-3}$.

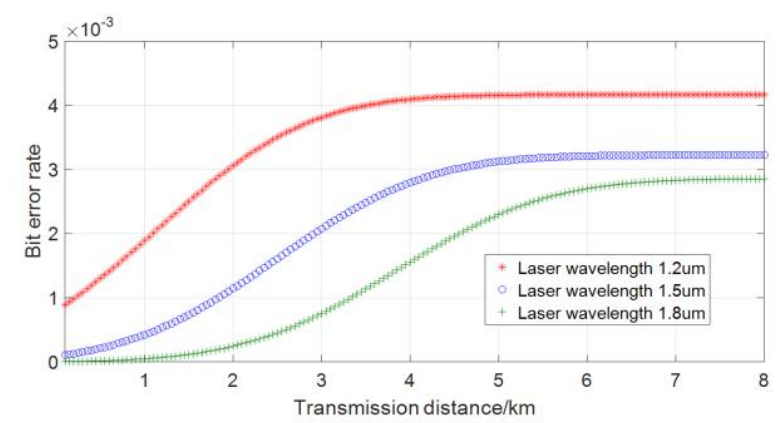

Figure 4. The curve of bit error rate and transmission distance under different laser's wavelength

Figure 4 illustrates that the bigger the laser's wavelength, the smaller the bit error rate. When the laser's wavelength is $1.8 \mu \mathrm{m}$, the transmission distance is $5 \mathrm{~km}$ and bit error rate is about $2.2 \times 10^{-3}$.

To ensure a certain bit error rate, we can increase wavelength by using multi-wavelength of laser, reduce the bit error rate, and effectively improve the transmission distance of atmospheric laser communication system.

According to formula (17), assuming that the atmospheric transmission channel has atmospheric attenuation effect and atmospheric turbulence effect, atmospheric attenuation and atmospheric turbulence respectively occupy a certain weighting in the atmospheric transmission channel. We quantitative analyze the different weighting of atmospheric attenuation effect and atmospheric turbulence effect that bring about the bit error rate. Figure 5 shows the curve of bit error rate of atmospheric laser communication system when the weighting of atmospheric attenuation is 0.2 and the weighting of atmospheric turbulence is 0.8 , when the weighting of atmospheric attenuation is 0.5 and the weighting of atmospheric turbulence is 0.5 , or when the weighting of atmospheric attenuation is 0.8 and the weighting of atmospheric turbulence is 0.2 .

The bit error rate of atmospheric laser communication system is not only related to the atmospheric attenuation effect and the atmospheric turbulence effect, but also related to the weighting of atmospheric attenuation effect and the weighting of atmospheric turbulence effect. If the transmission distance is $5 \mathrm{~km}$, the weighting of atmospheric attenuation is 0.2 , the weighting of atmospheric turbulence is 0.8 and the bit error rate is about $3.8 \times 10^{-3}$. But the weighting of atmospheric attenuation is 0.8 , the weighting of atmospheric turbulence is 0.2 , and the bit error rate is about $1.5 \times 10^{-3}$. Through quantitative calculations and analysis, we can observe that the bigger the weighting of atmospheric turbulence, the higher the bit error rate, and the atmospheric turbulence has greater impact. But, we cannot ignore the effects of atmospheric attenuation. Considering the influence of atmospheric attenuation and atmospheric turbulence, we establish the model of bit error rate to obtain scientific 
data of bit error rate. The main reason is that the model can truly make the bit error rate in the actual laser communication system known.

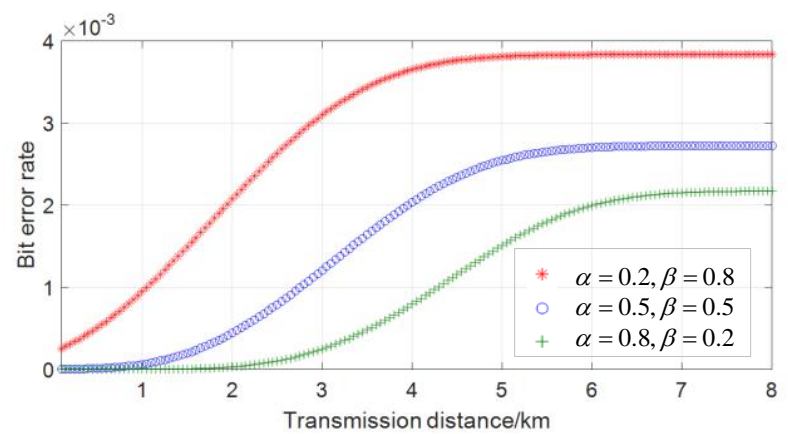

Figure 5. The curve of bit error rate of atmospheric laser communication system

\section{Conclusions}

This paper analyses the influencing factors of atmospheric laser communication system, under the atmospheric attenuation effect. We give the calculation model of atmospheric transmittance and transmission distance and atmospheric visibility, and establish the correlation function of bit error rate and transmission distance and atmospheric visibility and laser intensity. Under the atmospheric turbulence effect, according to the logarithmic variance of intensity's fluctuation, we obtain the relationship between the probability density function of light intensity and the signal to noise ratio of photoelectric detector. We set up the relation of bit error rate and the logarithmic variance of intensity's fluctuation, and analyze the relation of the bit error rate and the intensity of atmospheric turbulence and the wavelength of laser. Considering the atmospheric attenuation effect and the atmospheric turbulence effect simultaneously exist in the atmospheric channel, we deduce the mathematical model of bit error rate of atmospheric laser communication system. This paper establishes the model of bit error rate to improve the accuracy of transmission information of atmospheric laser communication system, and provides analysis basis for laser communication system.

\section{Acknowledgements}

This work has been supported by the Key Programs of Shaanxi science and Technology Department (No. 2017JM6041).

\section{References}

1. C. Y. Chen, H. M. Yang, H. L. Jiang, "Performance analysis of large-aperture receiving and selection of aperture size in atmospheric optical communications," Chinese Journal of Lasers, vol. 36, no. 11, pp. 2957-2961, 2009

2. R. Dashen, "Path integrals for waves in random media," J.Math.Phys, vol. 20, no.5, pp. 894-920, 1979

3. Z. H. Gao, B. D. Lv, "Partially coherent nonparaxial Hermite-Caussian beams and their propagation properties," Optic, vol. 118, pp. 307-314, 2007

4. H. S. Li, "Limited magnitude calculation method and optics detection performance in a photoelectric tracking system," Applied Optics, vol. 54, no. 7, pp. 1612-1617, 2015

5. H. S. Li, D. Pan, "Multi-photoelectric detection sensor target information recognition method based on D-S data fusion," Sensors and Actuators A-Physical, vol. 264, pp.117-122, 2017

6. B. D. Lv, K. L. Duan, “Nonparaxial vectorial,” Infrared and Laser Engineering, vol. 35, no. 2, pp. 127-129, 2006

7. D. F. Wang, Z. F. Chu, Z. L. Ren, G. D. Li, "Influence of atmospheric turbulence on BER of laser communication system," Laser \& Infrared, vol. 41, no. 4, pp. 390-393, 2011

8. J. G. Wang, Y. J. Zhao, D. Chen, Y. H. Wang, "Effects of turbulence sizes on the error rate of atmospheric laser communication system," Infrared and Laser Engineering, vol. 38, no. 4, pp. 655-659, 2009

9. X. J. Wu, H. X. Wang, M. Liu, "Affect analysis of receiving aperture on bit-error rate performance in free space optical communication systems under different visibilities," Acta Optica Sinica, vol. 33, no. 10, pp. 47-51, 2013

10. X. Yi, P. Yue, Z. J. Liu, G. Chen, "SER performance analysis of radio over free space optical systems," Journal of Optoelectronics Laser, vol. 23, no. 7, pp. 1309-1313, 2012

11. Y. X. Zhang, S. Yin, "Effects of the scale of turbulence on optical resolution of imaging system in turbulence," Chinese journal of lasers, vol. 27, no. 7, pp. 655-659, 2000

12. Z. G. Zhao, B. D. Lv, "Partially coherent vectorial cosh-Gaussian beams beyond the paraxial approximation," Opt Commun., vol. 270, pp.8-15, 2007 